\title{
Optimization of discrete Raman amplifiers for different kinds of fibers
}

\author{
J.D.Ania-Castanon and S.K.Turitsyn \\ Photonics Research Group, Aston University, \\ Birmingham B4 7ET, UK
}

\begin{abstract}
We present an analysis of the performance of backward-pumped discrete Raman amplifier modules designed for simultaneous amplification and dispersion and/or dispersion slope compensation, both in single-channel and in multichannel systems. Optimal module parameters are determined within a realistic range of pump and signal powers.
\end{abstract}

Keywords: Raman amplifiers, dispersion compensation

\section{INTRODUCTION}

The advent of high-power pump lasers has made possible the commercial application of Raman amplifiers (RAs) in optical communications ${ }^{1-6}$. With an appropriate selection of the gain medium, discrete Raman amplifiers can provide both amplification and dispersion compensation over a large bandwidth, thereby simultaneously overcoming two major limiting factors in high-bit rate transmission. The advanced discrete RA module presented in ${ }^{6}$ can compensate for the loss, dispersion and slope of $49 \mathrm{~km}$ of SMF. By varying the gain medium of the discrete RA, it is possible to achieve simultaneous amplification and dispersion compensation for different types of transmission fiber. Obviously, there is a certain trade-off between the RA gain and its capability to compensate for dispersion. Design of the optimal amplifying/compensating module also depends on the pump and input signal powers. Therefore, careful optimization of the module parameters is required to achieve both efficient amplification and dispersion compensation. Because of the variety of available configurations, it is not practical to study all possibilities experimentally, and accurate numerical modelling plays a crucial role in the design and optimization of such modules for dispersion compensation and Raman amplification.

In this paper, we present numerical design analysis of a range of discrete Raman amplifiers, considering three basic gain media: Single-Mode Fiber (SMF), Dispersion Compensating Fiber (DCF), Dispersion Shifted Fiber (DSF), and their combinations. Using this fiber base, we perform a comparative analysis of the following three main types of amplifier module: (a) simultaneous compensator for anomalous, or (b) normal dispersion transmission span, and (c) discrete Raman amplifier with zero total dispersion of the module, with the possibility of slope compensation. We divide our study of the amplifier modules in two sections, focusing initially in their performance in single-channel operation, and then as part of a multichannel system.

Our analysis takes into account the effects of amplified spontaneous emission and pump depletion. Note that in the problem under consideration there is no appreciable third order Stokes generation, and this simplifies the modelling.

The optimization procedure aims: (1) to determine maximum possible gain delivered with a given pump and signal; and (2) to find the optimal region in which the amplifier can operate either as a device with zero dispersion, or as a simultaneous compensator for fiber losses and dispersion of a transmission fiber span. The present analysis is limited to the case of the backward-pumped Raman amplifiers.

*E-mail: aniacajd@aston.ac.uk 
Table 1: Summary of Fiber Parameters.

\begin{tabular}{|l|l|l|l|}
\hline & \multicolumn{1}{|c|}{ SMF } & \multicolumn{1}{c|}{ DCF } & \multicolumn{1}{c|}{ DSF } \\
\hline Loss $(1455)$ & $0.3 \mathrm{~dB} / \mathrm{Km}$ & $1.2 \mathrm{~dB} / \mathrm{Km}$ & $0.36 \mathrm{~dB} / \mathrm{Km}$ \\
\hline Loss $(1550)$ & $0.2 \mathrm{~dB} / \mathrm{Km}$ & $0.81 \mathrm{~dB} / \mathrm{Km}$ & $0.24 \mathrm{~dB} / \mathrm{Km}$ \\
\hline Loss $(1658)$ & $0.3 \mathrm{~dB} / \mathrm{Km}$ & $1.2 \mathrm{~dB} / \mathrm{Km}$ & $0.36 \mathrm{~dB} / \mathrm{Km}$ \\
\hline $\mathrm{g} / \mathrm{A}_{\text {eff }}(1550)$ & $0.4 \mathrm{Km}^{-1} \mathrm{~W}^{-1}$ & $1.8 \mathrm{Km}^{-1} \mathrm{~W}^{-1}$ & $0.8 \mathrm{Km}^{-1} \mathrm{~W}^{-1}$ \\
\hline $\mathrm{g} / \mathrm{A}_{\text {eff }}(1658)$ & $0.36 \mathrm{Km}^{-1} \mathrm{~W}^{-1}$ & $1.5 \mathrm{Km}^{-1} \mathrm{~W}^{-1}$ & $0.72 \mathrm{Km}^{-1} \mathrm{~W}^{-1}$ \\
\hline $\mathrm{D}(1550)$ & $17 \mathrm{ps} \mathrm{nm}^{-1} \mathrm{Km}^{-1}$ & $-90 \mathrm{ps} \mathrm{nm}^{-1} \mathrm{Km}^{-1}$ & $0 \mathrm{ps} \mathrm{nm}^{-1} \mathrm{Km}^{-1}$ \\
\hline $\mathrm{S}(1550)$ & $0.058 \mathrm{ps} \mathrm{nm}^{-2} \mathrm{Km}^{-1}$ & $-0.3 \mathrm{ps} \mathrm{nm}^{-2} \mathrm{Km}^{-1}$ & $0.02 \mathrm{ps} \mathrm{nm}^{-2} \mathrm{Km}^{-1}$ \\
\hline
\end{tabular}

\section{SINGLE CHANNEL OPERATION}

The length of the amplifier is defined here as optimal when the overall gain (the ratio of the output power to the input signal power) reaches its maximum. Fig. 1 depicts the variation of the gain with the change of the length of fiber for five different amplifier gain media: pure SMF, zero-dispersion DSF, pure DCF and zero-average dispersion fiber combinations DCF+SMF, and SMF+DCF. The figure shows that the maximum gain is highest in the DSF, followed by the DCF, SMF+DCF, DCF+SMF and SMF configurations, in that order.

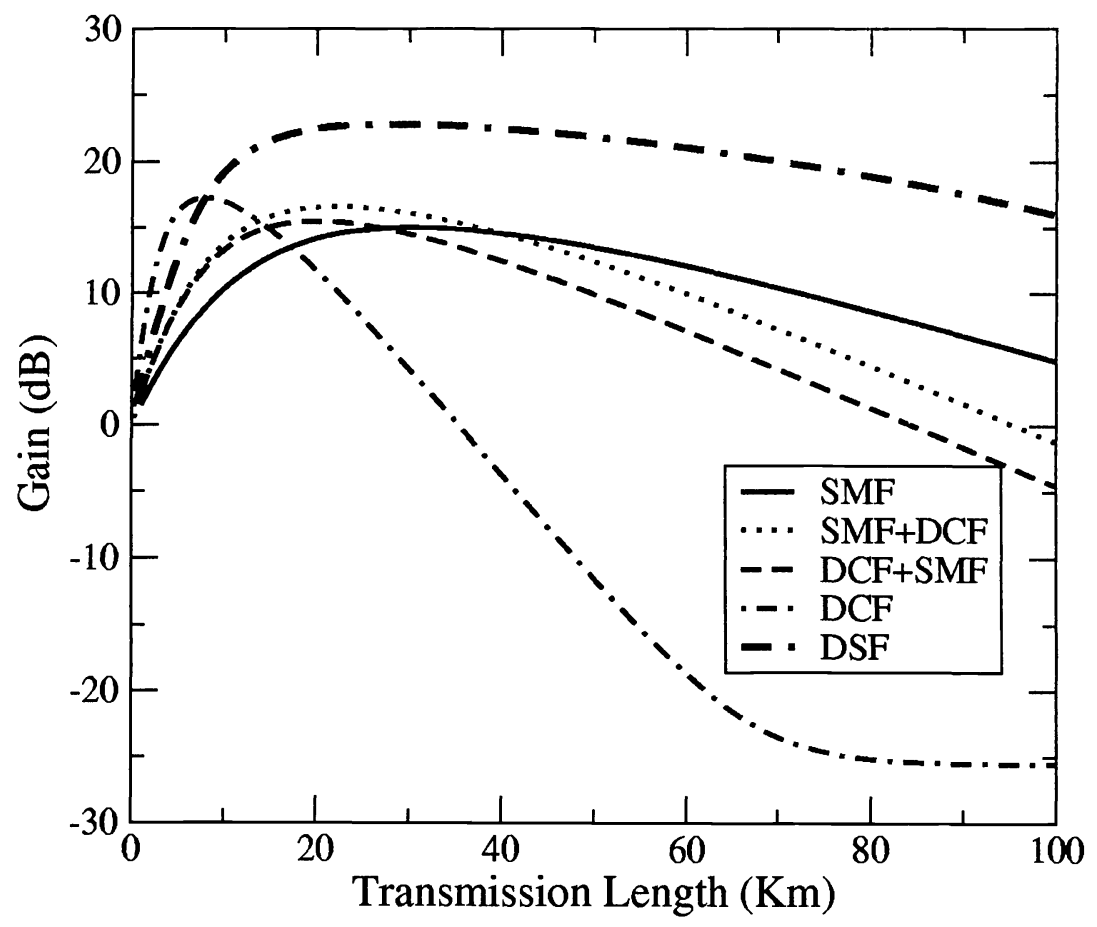

Figure 1: Gain vs. Transmission Length for five different media. 
For short amplifier lengths, the gain is higher in the DCF configuration, but the high loss leads to a fast decay with further increase of the amplification length. Note that the gain is better maintained for longer distances in SMF than in any configuration that includes DCF. This is an important factor for consideration of the amount of dispersion that can be compensated by an amplifier module. The dependence of the maximum gain and the optimal amplifier length on the input signal power for the five configurations under consideration is shown in Fig. 2.
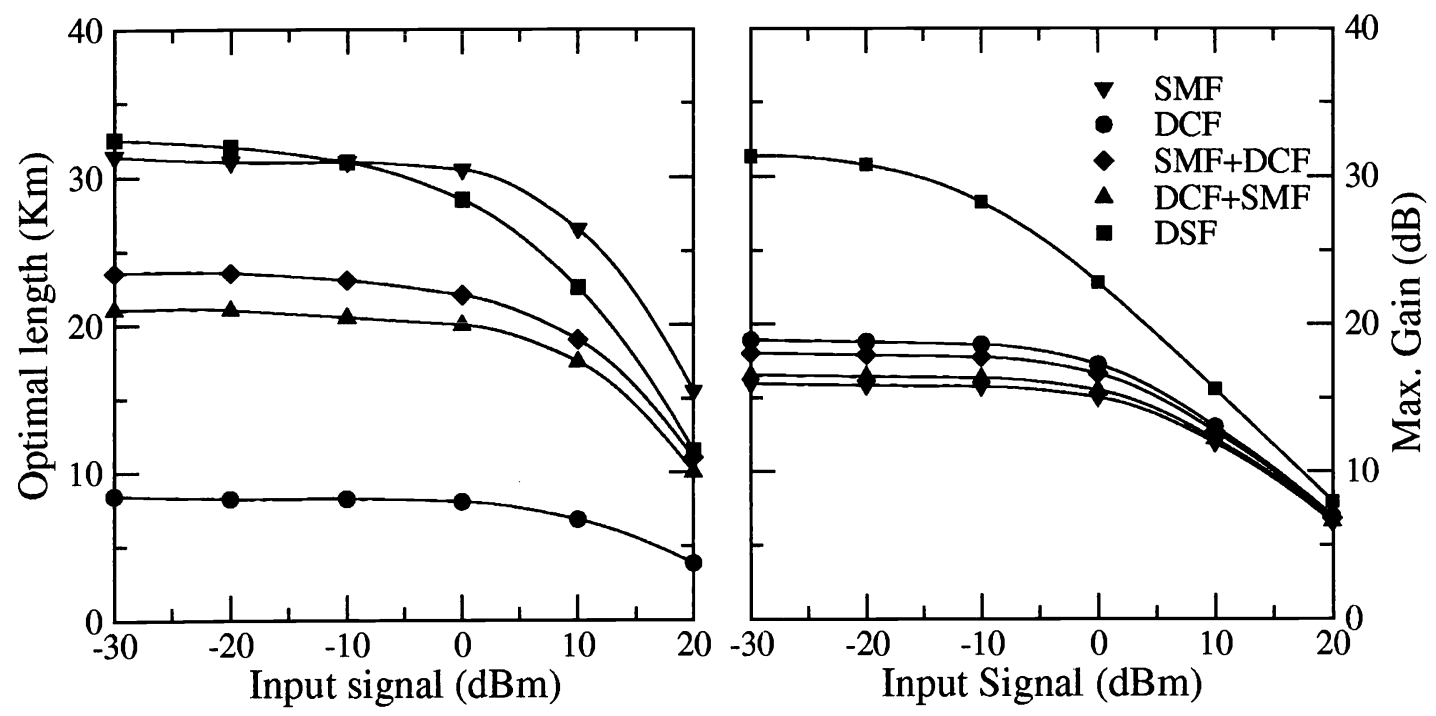

Figure 2: Optimal length and gain vs. input signal power.

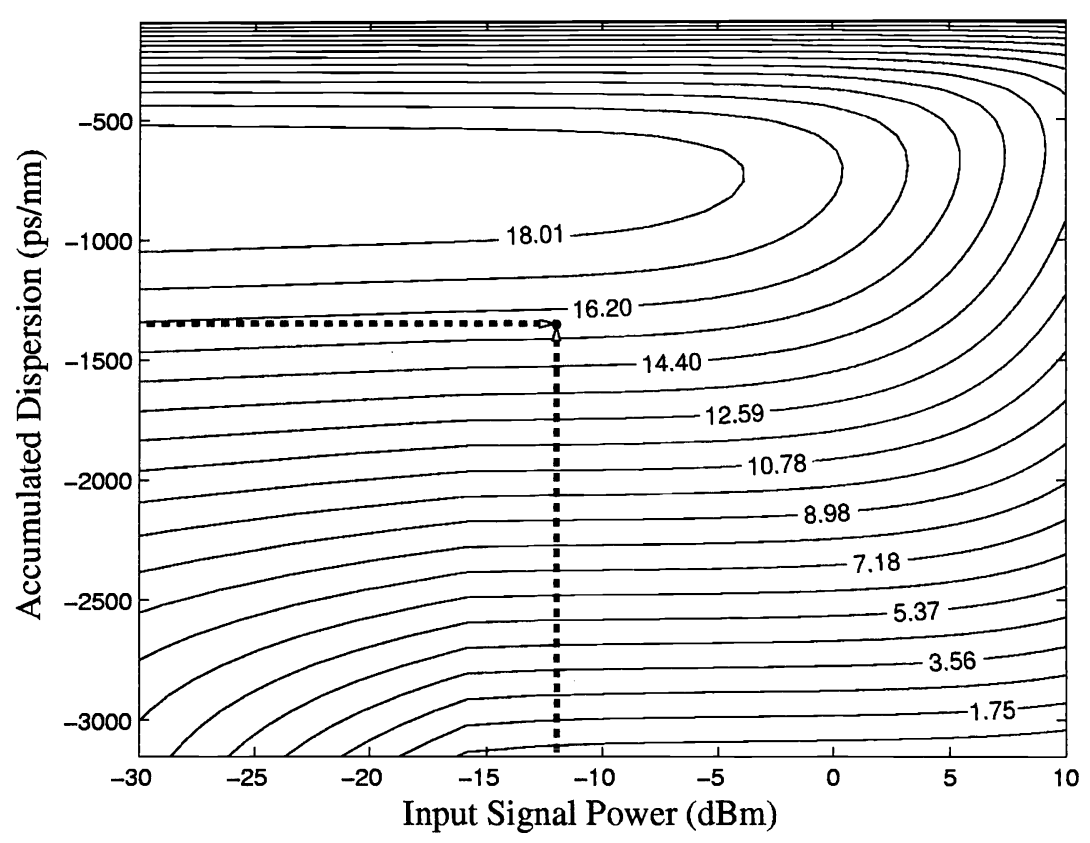

Figure 3: Gain in the DCF with $1 \mathrm{~W}$ fixed pump power and different input signals.

It is important to analyse the correlations between achievable gain and the amount of accumulated dispersion for different pump powers and input signal powers. The latter parameter is obviously important, as it determines the transmission span length after which the module can be inserted with the maximal effect. Figure 3 plots the variation of 
the amplifier gain against the input signal power and the accumulated dispersion in the transmission link in the case of pure DCF. The dashed lines show a simple design rule. To compensate for a specific amount of dispersion one should follow the horizontal line until it crosses the curve with the desired total gain. The vertical line then specifies an appropriate input signal power as in Fig. 3) or the required pump power, as in Fig. 4. The fast decay of the gain in the DCF link limits the optimal region of operation in which the amplifier effectively delivers dispersion compensation and a high gain at the same time. Using a DCF-based amplifier module to compensate for the dispersion accumulated in a $100 \mathrm{~km}$ SMF span $(1700 \mathrm{ps} / \mathrm{nm})$ with a $1 \mathrm{~W}$ pump and an input signal below $-10 \mathrm{dBm}$, a gain higher than $12 \mathrm{~dB}$ can be demonstrated. On the other hand, with the same pump power we can achieve full dispersion, slope and loss compensation for the case of a $4 \mathrm{dBm}$ signal propagated through $78 \mathrm{~km}$ of SMF (signal at the amplifier input $<-11.6 \mathrm{dBm}$, dispersion $1326 \mathrm{ps} / \mathrm{nm} / \mathrm{km}$ ). Full compensation of both the dispersion and the loss generated in a 100-km SMF link requires a higher power for the pump, i.e. $1.37 \mathrm{~W}$ for the case of a $1 \mathrm{~mW}$ signal. Gains over $16 \mathrm{~dB}$ for input signals of 0 $\mathrm{dBm}$ or less can be obtained using a $1 \mathrm{~W}$ pump over a range of 400 to $-1400 \mathrm{ps} / \mathrm{nm}$ of accumulated dispersion. Figure 4 demonstrates that using a SMF-based module (to compensate for the normal dispersion), a gain $>12 \mathrm{~dB}$ is available for a $0 \mathrm{dBm}$ signal with pump powers close to $30 \mathrm{dBm}$. The same holds true for any input signal between -20 and $10 \mathrm{dBm}$, and an accumulated dispersion between 300 and $1100 \mathrm{ps} / \mathrm{nm}$. The maximum gain, obtained for the lower power signals, is about $16 \mathrm{~dB}$.

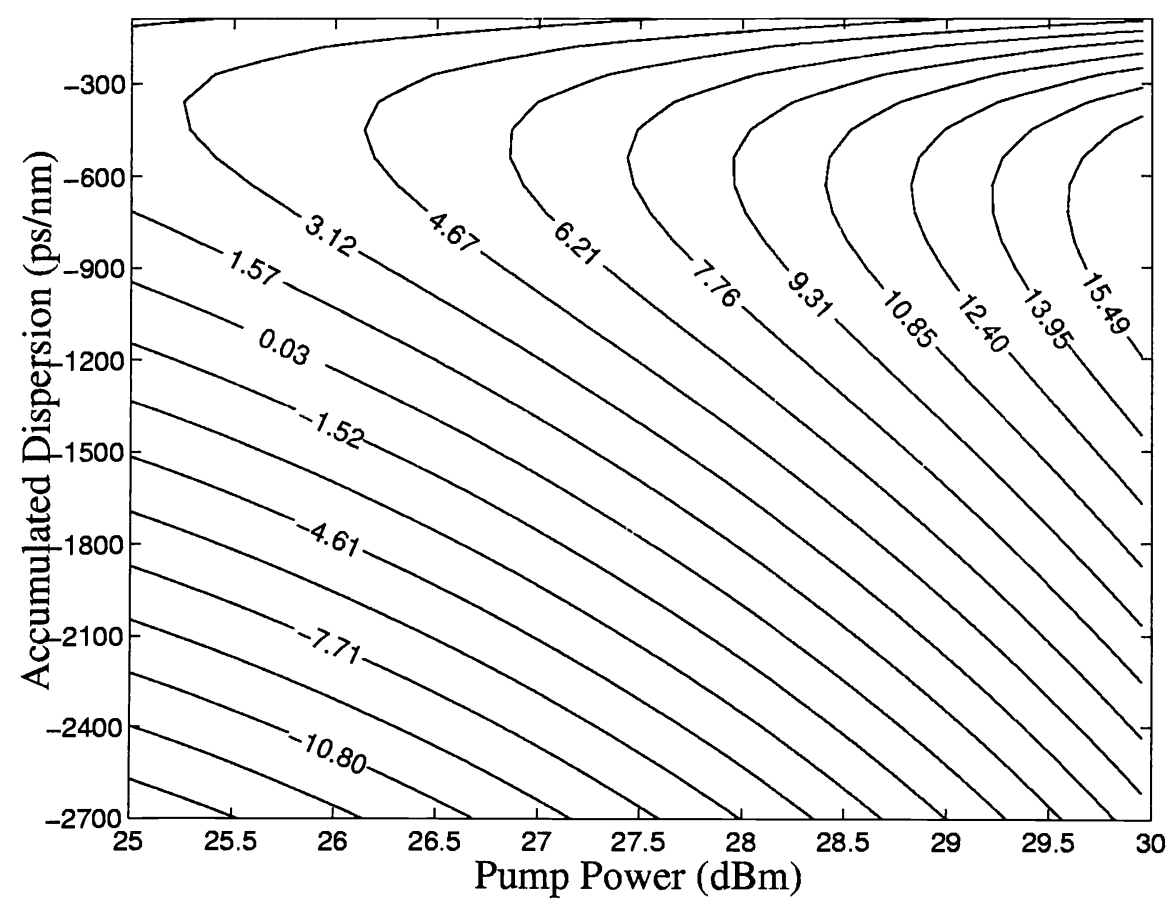

Figure 4: Gain in the DCF with $1 \mathrm{~mW}$ fixed input signal and variable pump power

The use of DCF modules as active Raman media, though advantageous in terms of total gain, results in a higher incidence of double Rayleigh scattering in the total noise performance. This problem is greatly reduced when the core area of the gain medium is broader, as in the case of SMF. Under certain circumstances it can be interesting, then, to pump directly the SMF fiber while using the DCF module only for dispersion compensation, while maintaining a total zero average dispersion of the fiber link. Figures 5 and 6 plot the variation of the amplifier gain as a function of the signal power and the accumulated dispersion, and against pump power and accumulated dispersion, respectively, for the case of SMF. Again, this two figures can be used for simple design guidelines. By displacing ourselves through an horizontal line at the desired accumulated dispersion in Fig. 5 until we reach our signal power, we can obtain the total gain of the amplifier at a fixed pump power (of $1 \mathrm{~W}$, in this case). 
With the same procedure, for a fixed signal power, we can know from Fig. 6 the desired pump power to obtain a given gain with a specific accumulated dispersion.

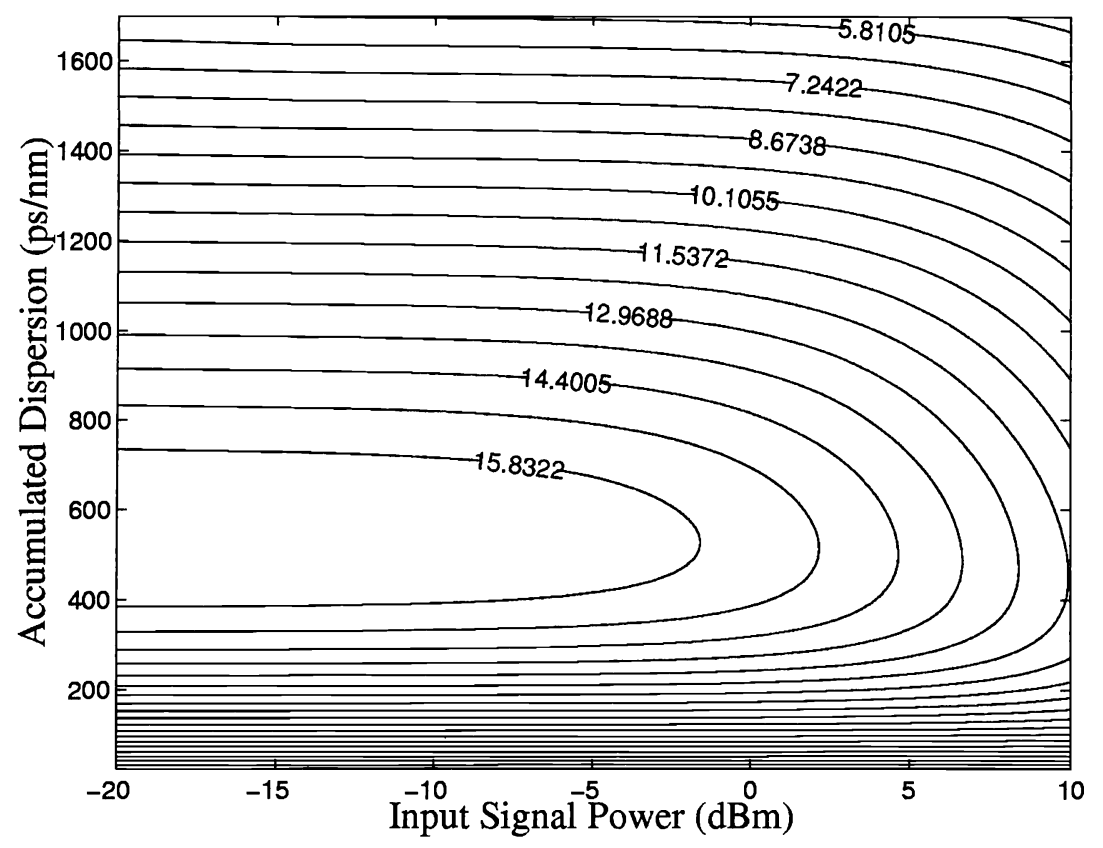

Figure 5: Gain in the SMF with $1 \mathrm{~W}$ fixed pump power and different input signals

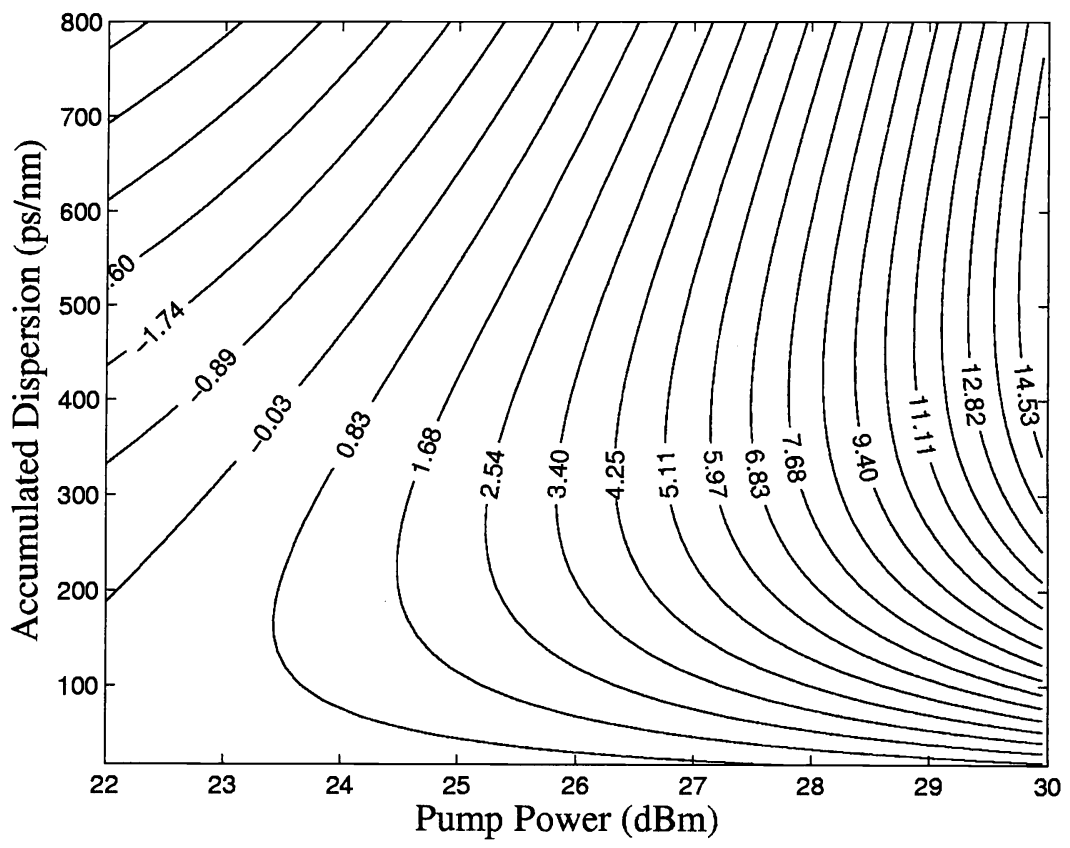

Figure 6: Gain in the SMF module with $1 \mathrm{~mW}$ fixed input signal and different pump powers 


\section{MULTI-CHANNEL OPERATION}

We now study the impact on the performance of the amplifier modules of the inclusion 8 WDM channels. The channels are separated $90 \mathrm{GHz}$, and symmetrically distributed both sides of the Stokes frequency of the pump, so the gain coefficient for all them can be considered the same up to the second decimal. The inclusion of multiple channels increases the total power of the signal, thus causing faster pump depletion, and the total noise bandwidth.

Figure 7 shows the variation of the optimal length and maximum gain against input power for each of the original configurations of amplifier modules, again for a fixed pump power of $1 \mathrm{~W}$. By comparison with Fig. 2 we can see that at high signal powers, pump depletion strongly affects the performance of the amplifier modules, reducing the maximum gain attainable for each channel. As expected, the DSF module is still the one that provides a higher potential gain, but the distance at which this maximum gain is obtained decreases faster with the increase of the input signal power, so that for any power above $-20 \mathrm{~dB}$, the Optimal length is always higher in SMF.
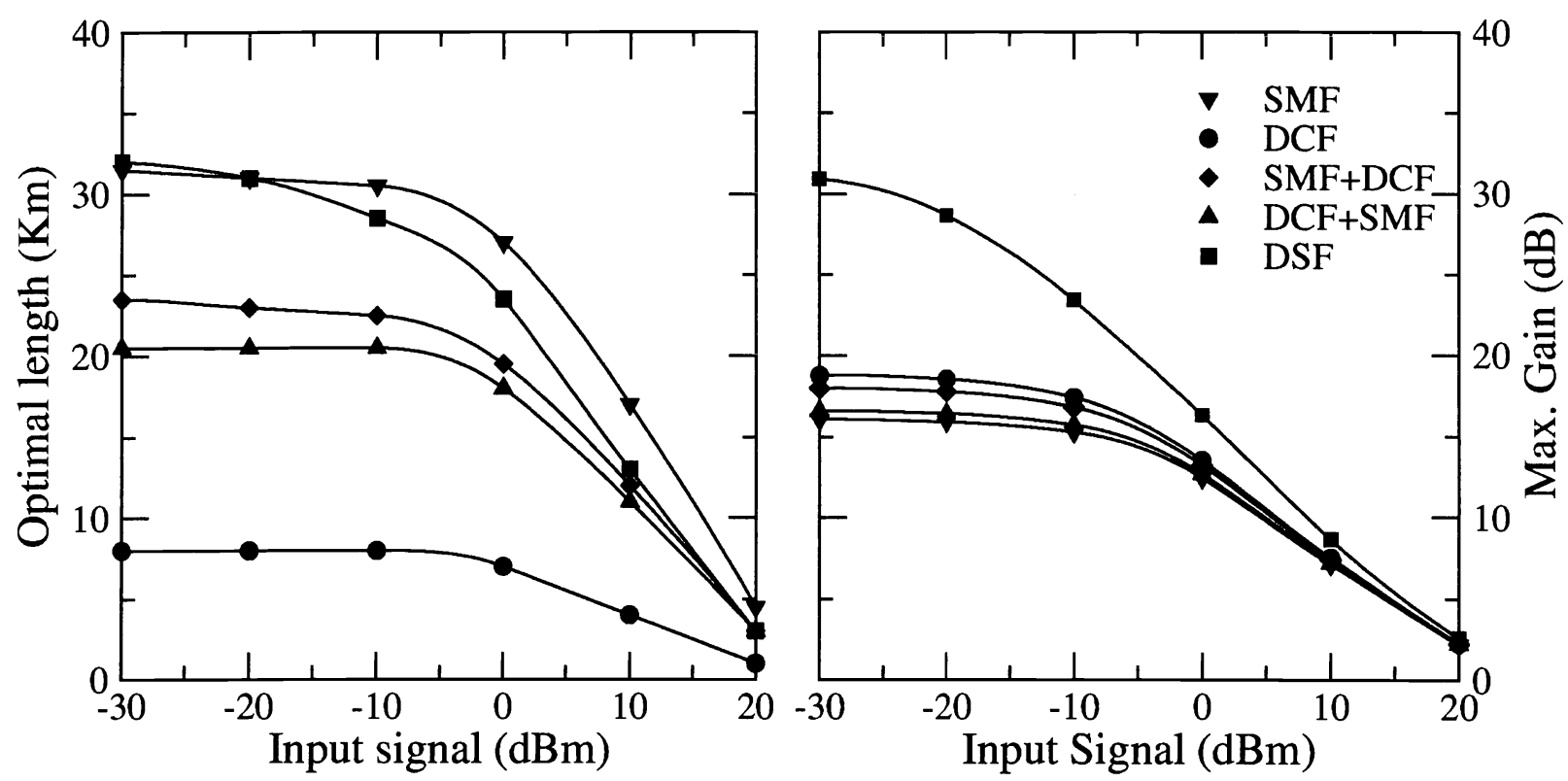

Figure 7: Optimal length and gain vs. input signal power in the multi-channel configuration

The effect of pump depletion is much less important when the signal powers are low, as it will usually be the case at the input of the amplifier module. Figure 8 compares the performance of a $-1700 \mathrm{ps} / \mathrm{nm}$ DCF amplifier module, designed to compensate for the loss and dispersion in $100 \mathrm{~km}$ of SMF, in both the single-channel and the multichannel situations. Section 8a depicts the dependency of the gain on the pump power for both cases. The dotted line represents the ideal gain for compensating at the same time for loss and dispersion in the SMF link. The signal input power is in this case $16 \mathrm{dBm}$, corresponding to an original $4 \mathrm{dBm}$ signal that has been propagated through $100 \mathrm{~km}$ of SMF. In this case, the ideal pump power, of about $1260 \mathrm{~mW}$, is nearly identical in both cases. Figure 8b shows how the gain varies with the signal power when the pump power is fixed, in this case to the previously ideal value of $1260 \mathrm{~mW}$. Here we can see that for higher signals, the performance decays quite fast, but the gain in the case of 8 channels is still about $13 \mathrm{~dB}$ for a 0dBm signal. 

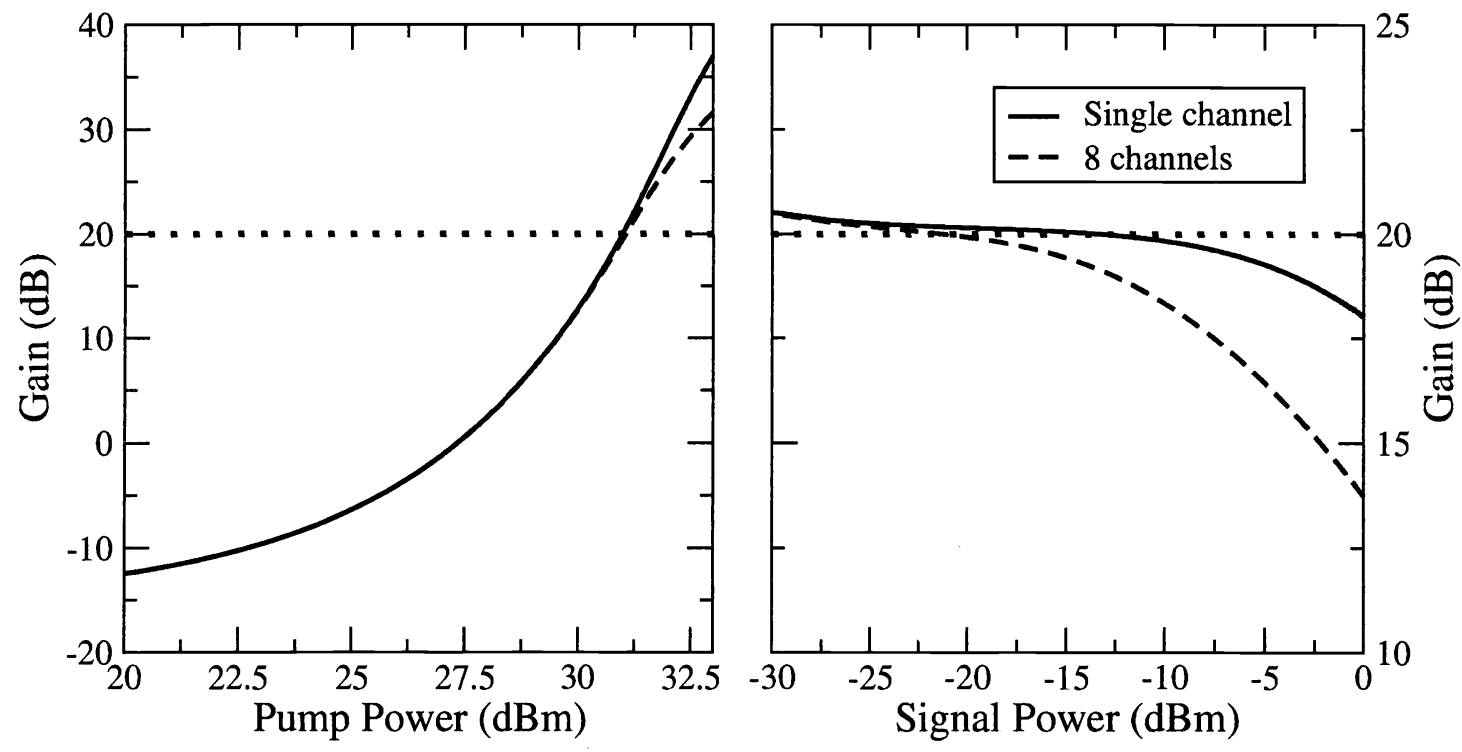

Figure 8: Comparison of single and multi-channel performances vs. signal and pump powers.

\section{CONCLUSIONS}

An analysis of the optimal parameters of backward-pumped discrete Raman amplifiers designed for simultaneous dispersion compensation and signal amplification using SMF and DCF has been presented, together with simple design rules for amplifying/compensating modules. Optimal amplifier lengths and gains have been determined within a realistic range of pump and signal powers for both single channel and 8-channel operation. Our DSF fiber, with both good Raman gain coefficient and limited loss, has proved to be ideal in terms of total gain and can be used for slope compensation, although combinations of SMF and DCF can also provide the basis for efficient zero-dispersion amplifiers. Under typical operating conditions in which the signal power at the amplifier module is low, the performance of single-pump multi-channel systems is similar to the obtained in the single channel case.

\section{REFERENCES}

1. S. Chernikov et al., OFC'99 Technical Digest, 1999, WG6.A.

2. Fludger et al., OFC'2000 Technical Digest, 2000, FF2.

3. A. Evans et al., OFC'2001 Technical Digest, 2001, MA7.

4. T. Okuno et al., IEEE Phot. Technol. Lett. 13, no. 8, 2001, 806-808.

5. L. Goldberg. et al., OFC'2002 Technical Digest, 2002, WJ7, pp. 251-262

6. T. Miyamoto et al., OFC'2002 Technical Digest, 2002, TuJ7, pp. 66-68. 